\title{
Avispas exóticas en la Patagonia: la importancia de la ecología de invasiones en el manejo de plagas
}

\author{
José M. Villacide ${ }^{1}$; Maité Masciocchi ${ }^{2, \bowtie} \&$ JuAn C. Corley $^{2}$ \\ 1. INTA. Grupo de Ecología de Poblaciones de Insectos. EEA Bariloche, Argentina. 2. CONICET. Grupo de Ecología de \\ Poblaciones de Insectos. INTA EEA Bariloche, Argentina.
}

\begin{abstract}
RESUMEN. El manejo integrado de plagas (MIP) se basa en el conocimiento de la ecología de las especies problema. Se ha sugerido que cuando las plagas son especies invasoras, la comprensión del proceso de invasión puede mejorar aun más nuestra capacidad de control de las plagas. Nuestro objetivo es revisar algunos atributos claves de la ecología y el comportamiento en el proceso de invasión de la avispa de los pinos Sirex noctilio y de la avispa chaqueta amarilla Vespula germanica, y discutir sus implicancias sobre las acciones de manejo implementadas en el N.O. de la Patagonia Argentina. Investigaciones previas identificaron los factores determinantes del éxito de invasión, como la demografía, los atributos de la comunidad receptora y el papel de los disturbios, tanto para estas especies invasoras como para otras. Aquí ampliamos ese conocimiento y nos enfocamos en aquellos aspectos ecológicos y de conducta característicos de las distintas etapas del proceso de invasión. Mientras las acciones de control para la avispa de los pinos tuvieron carácter regional y forman parte de un plan de manejo establecido, para la chaqueta amarilla las medidas fueron de carácter local y sin coordinación espacio-temporal. En ambos casos, y pese a las acciones ejecutadas, las especies avanzaron geográficamente, se establecieron con éxito y generaron impactos importantes en nuevas áreas. Sugerimos que los limitados alcances en términos de eficiencia de control de las poblaciones son, en parte, consecuencia de acciones diseñadas e implementadas con una escasa contemplación de los atributos ecológicos y comportamentales clave de estas especies.
\end{abstract}

[Palabras clave: Sirex noctilio, Vespula germanica, avispas invasoras, Hymenoptera, manejo integrado de plagas]

\begin{abstract}
Aвstract. Non-native wasps in Patagonia: the importance of Invasion Ecology in pest management: Integrated pest management (IPM) relies on thorough ecological knowledge of the targeted pests. It has been suggested that when these are non-native species, information on their invasion ecology could improve our abilities to successfully manage pest populations. Our aim is to revise the invasion history of two exotic wasps that have invaded NW Patagonia, and critically review control actions that have been and are implemented to manage their populations. Some critical factor affecting invasion success of the wood-wasp Sirex noctilio and the German wasp Vespula germanica have been previously investigated. Here, we extend such work, and focus on those ecological and behavioural aspects, relevant to each of the different stages of the invasion process. While most activities carried out to manage woodwasp populations are held at a regional level (covering the invaded habitats) and as part of specific management plan for the German wasp, actions are local and lack any spatial or temporal coordination. In both cases, despite the control activities implemented, regional spreads of the species have increased and populations established in new areas. We suggest that the limited success in control of these wasps could have been circumvented by considering both species not only as pests but as successful invaders.
\end{abstract}

[Keywords: Sirex noctilio, Vespula germanica, invasive wasps, Hymenoptera, pest control, IPM]

\section{INTRODUCCIÓN}

Las invasiones biológicas son uno de los problemas ambientales más serios con los que nos confrontamos en la actualidad. Como consecuencia del movimiento creciente de bienes y personas entre sitios y regiones, la presión de transporte de propágulos de especies desde sus áreas de origen y hacia ambientes nuevos ha aumentado de manera notable (Lockwood et al. 2007). Este proceso se refleja en el aumento constante del número de especies exóticas introducidas de modo deliberado o accidental en nuevos hábitats (Hulme 2009; Simberloff 2009).
Debido al desacople evolutivo de las especies exóticas en los sitios invadidos y a los procesos poblacionales característicos que operan cuando las densidades son bajas (i.e., efectos Allee), sólo una fracción pequeña de las especies que arriban a sitios nuevos logra establecerse y sostener poblaciones en niveles capaces de generar impactos notorios (Lockwood et al. 2007; Liebhold \& Tobin 2008; Davis 2009). Sin embargo, esa pequeña proporción es suficiente para considerar a las especies invasoras como una de las principales amenazas tanto para los ecosistemas naturales como para los productivos del planeta. Estas Última versión: 3 de enero de 2014; Aceptado: 3 de enero. 
especies pueden generar desequilibrios en la biodiversidad local, alteraciones en los servicios ambientales o provocar pérdidas significativas en la productividad de sistemas agro-forestales (Wingfield et al. 2008; Langor et al. 2009; Aukema et al. 2011; Liebhold et al. 2012). Por estas razones, muchas de las especies más conocidas por sus impactos notorios son especies invasoras (e.g., el pulgón de las cupresáceas Cinara cupressi, el escarabajo asiático Anoplophora glabripennis, la acacia negra Acacia mearnsii y el cactus Opuntia stricta) (Lowe et al. 2004).

La invasión de una especie en un ambiente nuevo es un proceso secuencial que puede ser descripto sobre la base de mecanismos que definen con claridad tres etapas (Lockwood et al. 2007; Liebhold \& Tobin 2008). El arribo, la etapa inicial del proceso en la cual los individuos son transportados fuera de su rango de distribución nativa hacia nuevas áreas, se encuentra determinado principalmente por el traslado antrópico de propágulos (e.g., semillas, esporas, huevos, larvas o reproductivos en latencia). Por su parte, el establecimiento es una etapa crítica en el proceso de invasión, en la cual las poblaciones son típicamente pequeñas y deben incrementar sus tamaños hasta niveles tales que minimicen los riesgos extinción local. En tanto, la expansión - fase del proceso en la cual las poblaciones incrementan su rango de ocupación geográfica en el área de invasiónestá determinada por la combinación de mecanismos dispersivos y reproductivos propios de la especie. Mientras que durante la fase de arribo no intervienen procesos poblacionales, el éxito de establecimiento y expansión en un ambiente no-nativo dependen fuertemente de rasgos propios de la historia de vida y de la dinámica poblacional de las especies, así como de los atributos de la comunidad receptora.

El éxito de los programas de manejo de especies invasoras depende de manera importante del diseño e implementación de estrategias que contemplen acciones específicas según la etapa del proceso de invasión (Liebhold \& Tobin 2008). Durante el arribo, las estrategias se basan en acciones de prevención (e.g., vigilancia y cuarentenas en zonas críticas) y tienen como fin evitar o minimizar el riesgo de ingreso de nuevas especies. La elección e implementación de estas acciones son dirigidas por análisis de riesgos, realizados sobre la base de un conocimiento detallado de las rutas y vías que promueven el movimiento de las especies. Durante el establecimiento, las acciones se centran sobre la detección precoz y la erradicación de los focos de invasión incipientes. A pesar de que el concepto de erradicación ha sido algo controversial en Ecología (ver Myers et al. 1998; Simberloff 2001), su papel es revisado en el diseño de las medidas de manejo del establecimiento de especies exóticas (Liebhold \& Tobin 2008). La erradicación -definida como la eliminación total de los individuos en un área determinada- es posible por ejemplo, mediante la implementación de acciones que promueven un incremento de los efectos Allee hacia niveles que conduzcan (por estocasticidad demográfica) a la población residente a su extinción local. Finalmente, el manejo de la expansión geográfica considera acciones que generan efectos sobre la reproducción y/o sobre la dispersión de los individuos reproductivos, a fin de reducir la tasa de ocupación del territorio. Se proponen para esta etapa acciones que limiten los eventos dispersivos, en particular los de larga distancia (e.g., barreras sanitarias, cuarentenas locales o control biológico con patógenos), así como aquellas que promuevan reducciones en el crecimiento poblacional (e.g., control biológico con parasitoides o predadores, control mecánico o control químico).

En este trabajo revisamos las acciones implementadas en el N.O. de la Patagonia Argentina para el manejo las avispas invasoras Sirex noctilio, una especie de importancia económica para la producción forestal, y Vespula germanica, una especie de importancia sanitaria en ambientes urbanos y periurbanos. Una primera aproximación al estudio de la Ecología de la Invasión de estas especies fue presentada por Farji-Brener \& Corley (1998). En ese estudio se exploraron las causas demográficas y del ambiente relacionadas con el éxito de invasión de estos y otros dos himenópteros en la región (el abejorro Bombus ruderatus y la hormiga cortadora de hojas Acromyrmex lobicornis). Aquí, extendemos ese trabajo y nos concentramos en los aspectos ecológicos y de comportamiento relevantes de $S$. noctilio y $V$. germanica en las distintas etapas del proceso de invasión. Para estas especies, a diferencia de B. ruderatus y A. lobicornis, se han implementado acciones de manejo a través de distintas estrategias, tanto en la escala local como en la regional. Nuestro objetivo es analizar los resultados obtenidos en dichas 
acciones a la luz de la ecología de invasiones. Esperamos no sólo recordar la importancia de la comprensión cabal de las características ecológicas de las especies para su control, sino también destacar la importancia de analizar el problema considerando el proceso histórico de invasión.

\section{HistORIA DE LA INVASIÓN Y DE MANEJO DE SiREX NOCTILIO Y VESPULA GERMANICA EN LA PATAgONIA ARGENTINA}

La avispa de los pinos, $S$. noctilio (Hymenoptera: Siricidae), es una especie solitaria que se ha convertido en uno de los problemas sanitarios principales en la mayoría de las regiones forestadas con Pinus spp. del mundo (Carnegie et al. 2006; Hurley et al. 2007; Yemshanov et al. 2009; Dodds et al. 2010; Villacide \& Corley 2012). Su capacidad de daño sobre el recurso forestal es muy importante, pudiendo alcanzar durante las fases epidémicas mortalidades cercanas a $80 \%$ de los árboles. En la actualidad, a más de un siglo de la primera detección fuera de su área nativa (norte de África y Europa), su distribución abarca una amplia diversidad de escenarios eco-climáticos y productivos (Lantschner et al. 2014). En Argentina su establecimiento fue documentado a fines de 1985 en la provincia de Entre Ríos. Durante la década del '90 se registraron nuevos focos de invasión tanto en la región Centro como en la Patagonia y la Mesopotamia. En la Patagonia, el arribo fue detectado en la localidad de Dina Huapi (Río Negro) en cargamentos de madera infestada proveniente de la provincia de Buenos Aires. La avispa se dispersó rápidamente a través de la región, y al presente ha alcanzado un rango de distribución consistente con la distribución de las aéreas implantadas con Pinus spp., desde el norte de la provincia de Neuquén hasta el sur de la provincia de Chubut. A pesar de una alta fragmentación del paisaje (los pinos en la región son plantados en parches de tamaño variable principalmente sobre una matriz de estepa herbácea), las poblaciones de la avispa se han expandido a una tasa promedio de $17.7 \mathrm{~km} /$ año (Figura 1a). Estas tasas de ocupación de territorio es una de las más bajas en comparación a las registradas en otras regiones invadidas del hemisferio sur (Lantschner et al. 2014).

El manejo de S. noctilio en la Patagonia ha sido implementadoa escala regional pororganismos oficiales, principalmente mediante acciones de control biológico clásico y aumentativo [a través de avispas parasitoides y un nematodo parásito (Tabla1)]. Los parasitoides Ibalia leucospoides

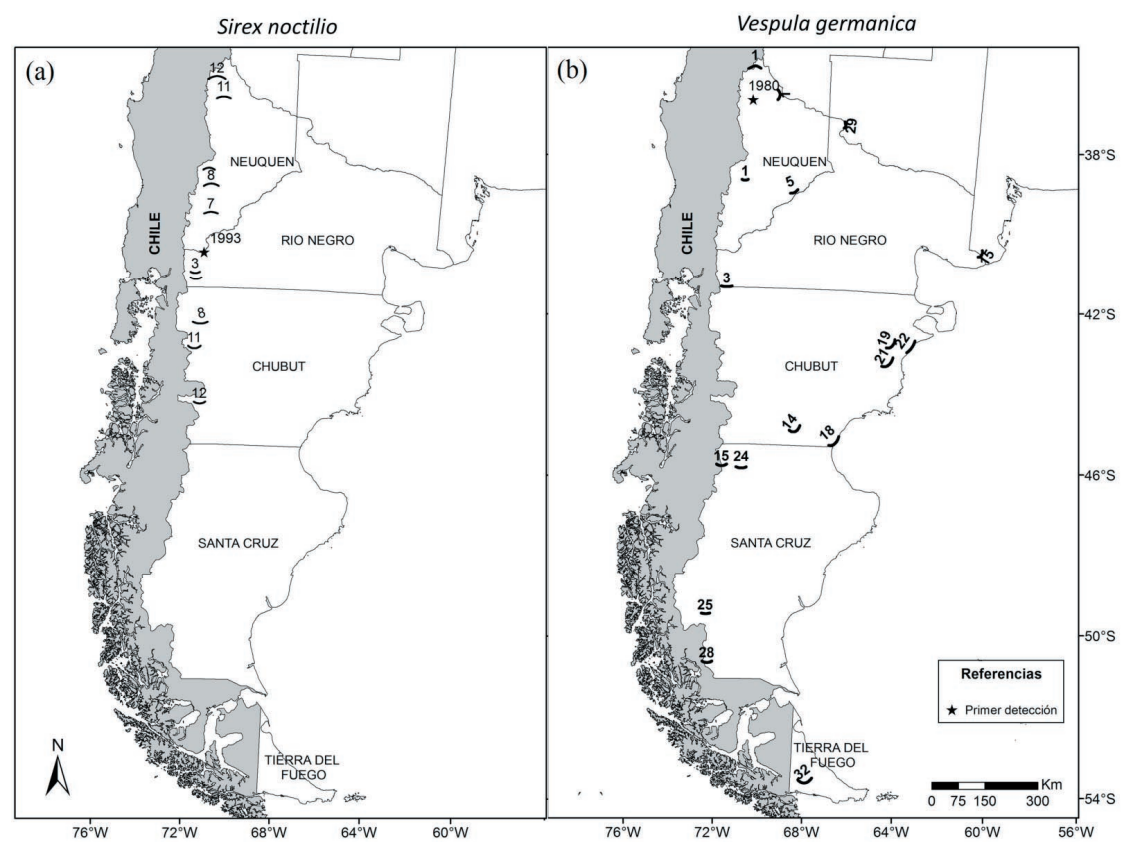

Figura 1. Mapa de distribución histórica de $S$. noctilio (a) y $V$. germanica (b) en la Patagonia argentina. Los números indican años trascurridos desde la detección original de la especie en la región.

Figure 1. Historical distribution map corresponding to S. noctilio (a) and V. germanica (b) in Patagonia. Figures denote the amount of years since the first report in the region. 


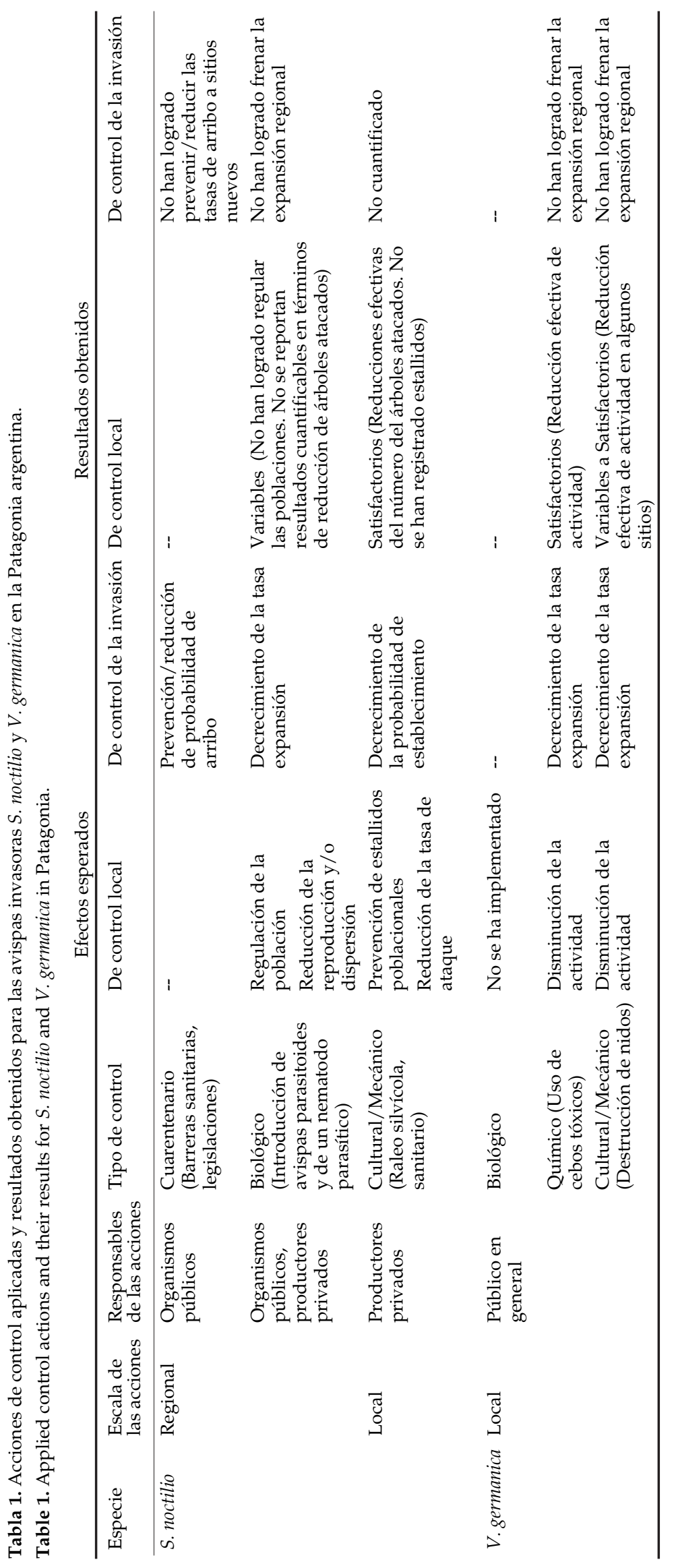


(Hymenoptera: Ibalidae) y Megarhyssa nortoni (Hymenoptera: Ichneumonidae), especies muy utilizadas en otras regiones invadidas por la plaga, han mostrado una elevada capacidad de establecimiento en la región (Villacide \& Corley 2003; Corley \& Bruzzone 2009). Ibalia leucospoides ha ingresado de modo accidental en conjunto con la plaga (probablemente mediante el transporte de madera infestada), y se ha distribuido a una alta velocidad a través de la región; ha alcanzado niveles de parasitismo de hasta $40 \%$ (Villacide \& Corley 2003, 2012). Aunque este parasitoide ha sido reconocido frecuentemente como exitoso, algunos aspectos propios (e.g., la interacción fisiológica con su huésped, la respuesta funcional o su capacidad de dispersión) sugieren que, en términos de regulación de las poblaciones de $S$. noctilio, su papel permanece poco claro (Bruzzone et al. 2009; Fischbein et al. 2011; Villacide \& Corley 2012). Por su parte, la avispa parasitoide $M$. nortoni, de introducción reciente en un número acotado de sitios, se ha dispersado hacia sitios cercanos, y ha alcanzado niveles de parasitismo que oscilan entre el 4 y el $70 \%$ (SENASA, datos no publicados). En tanto, el nematodo entomopatógeno Deladenus (=Beddingia) siricidicola, considerado a nivel mundial como el agente de control biológico más efectivo de $S$. noctilio (Lacey et al. 2001; Bedding \& Iede 2005), ha sido introducido con éxito en la región. Si bien los niveles de parasitismo registrado por este agente de control son en general altos, sus efectos en términos de reducción de la densidad local y/o prevención de los estallidos poblaciones de la plaga son aún dudosos (Hurley et al. 2007; Villacide \& Corley 2012).

En conjunto, las acciones de control biológico de S. noctilio implementadas en la Patagonia han mostrado resultados variables (Tabla 1 ). Si bien los enemigos naturales utilizados han mostrado una capacidad de establecimiento elevada y en general altos niveles de parasitismo, a escala local no han logrado regular ni reducir de manera significativa las densidades de las poblaciones de S. noctilio. Es importante notar que en plantaciones de la región sujetas a acciones de control biológico como las descritas, se han registrado estallidos poblacionales severos, con tasas de mortalidad de árboles superiores a $80 \%$. En tanto, a escala regional, y a pesar de un esfuerzo sostenido, la estrategia no ha logrado reducir o frenar la expansión geográfica de la avispa
(Lantschner et al. 2014; Corley et al. datos no publicados).

El control cultural de las plantaciones forestales, como estrategia de manejo de las poblaciones de la plaga, es una herramienta efectiva que permite prevenir y reducir su impacto local. El raleo sanitario, por ejemplo, ha demostrado ser una táctica de manejo eficiente en la reducción de las poblaciones de la avispa (Villacide \& Corley 2006). En esta línea, se ha sugerido que esfuerzos de extracción por sobre el $60 \%$ de los árboles infectados permitiría sostener la población de la plaga en niveles endémicos de bajo impacto, e incluso lograr la erradicación local. A pesar de su eficacia en el control de la plaga, las acciones de control basadas en el manejo de la plantación han sido implementadas de modo limitado (Villacide \& Corley 2006, 2012) (Tabla 1).

Vespula germanica es un véspido eusocial originario de Eurasia y del norte de África, que en la actualidad presenta una amplia distribución mundial (Archer 1998). Esta especie ha invadido con éxito Sudáfrica, Australia, Nueva Zelanda, América del Norte y América del Sur (Spradbery \& Maywald 1992; Tribe \& Richardson 1994; Archer 1998; D' Adamo et al. 2002). En el N.O. de la Patagonia fue observada por primera vez en 1980 en la localidad de Andacollo (provincia de Neuquén) a $30 \mathrm{~km}$ de la frontera con Chile (Willink 1980). Desde su detección hasta el presente se ha establecido en una diversidad amplia de hábitats, desde el norte de la provincia de Mendoza hasta el extremo sur de la provincia de Santa Cruz (Masciocchi \& Corley 2013). Recientemente fue detectada en la provincia Tierra del Fuego (A. Valenzuela, comunicación personal) (Figura 1b). La expansión geográfica registrada para $V$. germanica es una de las más altas observadas en himenópteros sociales invasores. En Argentina alcanza una tasa promedio de 37 $\mathrm{km} /$ año. Sin embargo, estudios recientes en condiciones controladas han mostrado que la capacidad propia de vuelo de las reinas es inferior a $2 \mathrm{~km}$. Esto sugiere que el transporte humano de propágulos ocultos (reinas hibernantes) es el principal factor que contribuye a su propagación geográfica (Masciocchi \& Corley 2013). V. germanica posee un potencial reproductivo importante (Tabla 2); la reina fecundada es la responsable del tamaño final de la colonia; en la época 
de mayor abundancia, esta colonia está conformada por miles de individuos.

El control de las poblaciones de $V$.germanica en la Patagonia ha sido eventual dado que no se ha declarado un plan específico ni para el manejo local ni para el regional (Tabla 1). Las acciones han sido implementadas por el público en general con diferentes métodos y sin una coordinación espacio-temporal adecuada. De todos los métodos de control propuestos para reducir las poblaciones de la avispa (ver detalles en Beggs et al. 2011), el control químico mediante cebos tóxicos ha sido el más eficiente a escala local (e.i., la eliminación de hasta el $99.7 \%$ de las colonias presentes en las áreas tratadas; Sackmann et al. 2001) y no requiere de la localización de los nidos para su aplicación. Sin embargo, a pesar de registrarse resultados satisfactorios en términos de reducción de la actividad de obreras en sitios determinados (Sackmann et al. 2001; Sackmann \& Corley 2007), estas acciones no han logrado efectos significativos sobre el control de la invasión de la especie (Masciocchi \& Corley 2013).

\section{ECOLÓGIA Y COMPORTAMIENTO: IMPLICANCIAS SOBRE EL MANEJO DE LA INVASIÓN DE S. NOCTILIO Y V. GERMANICA.}

En la Tabla 2 se resumen los principales aspectos de la ecología y el comportamiento de ambas especies según cada etapa del proceso de invasión. A pesar que algunos de ellos son reconocibles con facilidad como promotores del éxito de invasión, su contemplación en el diseño e implementación de los planes de manejo aplicados en la Patagonia ha sido escasa.

Para S. noctilio, la capacidad potencial de dispersión de adultos - de hasta $50 \mathrm{~km}$ en un día- (Bruzzone et al. 2009), así como el transporte humano de estadios inmaduros, no han sido considerados ni en la prevención del arribo hacia nuevas áreas ni en la implementación de barreras fitosanitarias o puntos de vigilancia. Por su parte, la implementación de estrategias locales de reducción de las poblaciones residentes de la avispa en áreas de establecimiento ha sido insuficiente en términos de intensidad y magnitud geográfica. Estas acciones pueden promover mecanismos que conduzcan a las poblaciones hacia densidades críticas para su supervivencia (Liebhold \& Tobin 2008). Las actividades implementadas para el manejo de $S$. noctilio en la Patagonia demuestran, por ejemplo, que la introducción del nematodo B. siricidicola no ha logrado desacelerar la tasa de expansión de $S$. noctlio a través del territorio forestado con pinos (Corley, datos no publicados). Asimismo, registros históricos muestran que, incluso luego de varios años de introducciones sucesivas de distintos enemigos naturales, la tasa de expansión regional de la avispa de los

Tabla 2. Aspectos ecológicos y comportamentales característicos de cada fase del proceso de invasión de S. noctilio y V. germanica.

Table 2. Ecological and behavioural features during the invasion process of S. noctilio y $V$. germanica.

\begin{tabular}{|c|c|c|c|}
\hline Fase de la invasión & $\begin{array}{l}\text { Atributo ecológico/ } \\
\text { comportamental }\end{array}$ & S. noctilio & V.germanica \\
\hline \multirow[t]{2}{*}{ Arribo } & Propágulos & $\begin{array}{l}\text { Ocultos (principalmente } \\
\text { estadios inmaduros) }\end{array}$ & Ocultos (reinas fecundadas) \\
\hline & Vías & Maderas infestadas & $\begin{array}{l}\text { Maderas, bienes domésticos, } \\
\text { vehículos }\end{array}$ \\
\hline \multirow[t]{3}{*}{ Establecimiento } & Plasticidad ecológica & Baja & Alta \\
\hline & Apareamiento & $\begin{array}{l}\text { Múltiple, antes de la } \\
\text { dispersión }\end{array}$ & $\begin{array}{l}\text { Múltiple, antes de la } \\
\text { dispersión }\end{array}$ \\
\hline & Desarrollo social & No presente, especie solitaria & Máximo, especie eusocial \\
\hline \multirow[t]{7}{*}{ Expansión } & Reproducción & & \\
\hline & Fenología & Ciclos anuales a tri-anuales & Ciclos anuales \\
\hline & $\begin{array}{l}\text { Potencial reproductivo } \\
\text { Dispersión }\end{array}$ & Muy alto & Alta \\
\hline & Capacidad de vuelo & Muy alta & Baja \\
\hline & Individuos dispersivos & Hembras y machos & Reinas \\
\hline & Mecanismos de re-distribución & $\begin{array}{l}\text { Vuelo/transporte de } \\
\text { propágulos }\end{array}$ & Transporte de propágulos \\
\hline & Dispersiones de larga distancia & $\begin{array}{l}\text { Frecuente, humano mediada, } \\
\text { movimiento propio }\end{array}$ & Frecuente, humano mediada \\
\hline
\end{tabular}


pino continuó aumentando (Villacide \& Corley 2012). En términos de reducción de la propagación geográfica, se podría esperar una mayor contribución del control biológico si el esfuerzo en la introducción repetida de enemigos naturales se centrara sobre el frente de avance de la plaga en lugar de en áreas con poblaciones establecidas en niveles endémicos o epidémicos (ver ejemplo de este tipo de estrategia en Sharov et al. 2002).

Vespula germanica es una especie con una plasticidad ecológica elevada (Harris 1996) (Tabla 2). La capacidad de las reinas fecundadas de hibernar en sitios protegidos, por ejemplo debajo de la corteza de maderas de uso doméstico y comercial, favorece el transporte accidental de la especie hacia nuevas áreas. En consecuencia, los mecanismos involucrados en el movimiento de los individuos reproductores de la especie reducen tanto la eficiencia de las acciones de manejo de las poblaciones establecidas como la prevención del arribo de nuevos reproductivos (en particular en regiones con numerosas vías de invasión). Por su parte, el manejo de las poblaciones incipientes durante la etapa de establecimiento (o durante la expansión) requiere de métodos sensibles para la detección precoz y la implementación de acciones tendientes a su erradicación local.

\section{CONSIDERACIONES FINALES}

A pesar de poseer historias evolutivas disímiles, las avispas $S$. noctilio y $V$. germanica han sido muy exitosas en la invasión de territorios nuevos, aun bajo condiciones ambientales severas y contrastantes (Beggs et al. 2011; Villacide \& Corley 2012). Desde el punto de vista básico, el éxito observado refleja la gran plasticidad ecológica y demográfica que han desarrollado estas especies (i.e., ajuste de su fenología a las condiciones ambientales locales, comportamiento reproductivo que reduce efectos Allee, mecanismos de dispersión de larga distancia por medios propios y a través de propágulos ocultos). Desde la óptica aplicada, la amplia distribución que las especies han alcanzado indica que su detección es compleja, en particular durante las fases iniciales de la invasión.

En la actualidad, el manejo integrado de plagas es la herramienta elegida para controlar las poblaciones de especies de insectosproblema dentro de un rango de valores tolerables, tanto a escala local como regional
(Wainhouse 2005). Mediante esta estrategia, que involucra el uso combinado y secuencial de varias acciones como el control químico, biológico y cultural, se busca minimizar el daño ocasionado por las plagas; a su vez, esto reduce el impacto ambiental de cada una de las acciones aplicadas por separado. En este contexto, el conocimiento de la biología de las plagas es clave para determinar el tipo, la intensidad y la secuencia de las acciones (Liebhold \& Tobin 2008; Villacide \& Corley 2012).

El manejo de especies invasoras plantea desafíos nuevos para el tradicional esquema de manejo integrado de plagas. En este contexto, se presenta una serie de factores críticos de consideración básica al momento del diseño de las acciones de manejo de la invasión. El análisis de riesgo de arribo y establecimiento, el desarrollo de métodos sensibles para la detección temprana, así como el conocimiento de la historia de invasión en otras regiones y de las características ecológicas y de conducta de las especies, son factores que, en conjunto, afectan la capacidad de manejo de las especies invasoras. De este modo, es importante que el esquema de manejo no sólo sea dinámico sino también que considere la ecología de la invasión en sentido amplio - los elementos que contribuyen al éxito de invasión - y mediante tecnologías validadas bajo las condiciones de los ambientes invadidos.

Agradecimientos: Este trabajo fue parcialmente financiado por los proyectos PIP-2010-11220090100043 (CONICET); PICT-1775 (Agencia para la Promoción Científica y Tecnológica), SaFo-109 Componente Plantaciones Forestales Sustentables (BIRF LN 7520 AR) y PNFOR 1104072 (INTA). Agradecemos MV Lantschner por su ayuda en la confección de los mapas.

\section{BIBLIOGRAFÍA}

ARCHER, ME. 1998. The world distribution of the euroasian species of Paravespula (Hymenoptera: Vespidae). Entomol. Mon. Mag., 134:279-284.

Aukema, JE; B Leung; K Kovacs; C Chivers; KO Britton; et AL. 2011. Economic impacts of non-native forest insects in the continental United States. PLOS ONE, 6:e24587.

Bedding, R \& E Iede. 2005. Application of Beddingia siricidicola for Sirex woodwasp control. En: Nematodes as biocontrol agents. Grewal, P; Ehlers, R \& Shapiro-Ilan, D (eds.). CAB International, Wallingford, U.K.

Beggs, JR; EG Brockerhoff; JC Corley; M Kenis; M MASCIOCCHI; ET AL. 2011. Ecological effects and management of invasive alien Vespidae. BioControl, 56:505-526.

Bruzzone, OA; JM Villacide; C Bernstein \& JC Corley. 2009. Flight variability in the woodwasp Sirex noctilio (Hymenoptera: Siricidae): an analysis of flight data using 
wavelets. J. Exp. Biol., 212:731-737.

Carnegie, AJ; M Matsuki; DA Haugen; BP Hurley; R Ahumada; et Al. 2006. Predicting the potential distribution of Sirex noctilio (Hymenoptera: Siricidae), a significant exotic pest of Pinus plantations. Ann. For. Sci., 63:119-128.

Corley, JC \& OA Bruzzone. 2009. Prolonged diapause and the success of parasitoids in biological control. Biol. Control, 51:471-474.

D' ADAMO, P; P SACKMANN; JC CORLEY \& M RabinOVICH. 2002. The potential distribution of German wasps (Vespula germanica) in Argentina. New Zeal. J. Zool., 29:79-85.

DAvIS, MA. 2009. Invasion biology. Oxford University Press, USA.

Dodds, KJ; P DE Groot \& DA Orwig. 2010. The impact of Sirex noctilio in Pinus resinosa and Pinus sylvestris stands in New York and Ontario. Can. J. Forest Res., 40:212-223.

FARJI-BRENER, AG \& JC CORLEY. 1998. Successful invasions of hymenopteran insects into NW Patagonia. Ecol. Austral, 8:273-249.

Fischbein, D; JC CoRley; JM Villacide \& C Bernstein. 2011. The influence of food and con-specifics on the flight potential of the parasitoid Ibalia leucospoides. J. Insect Behav., 24:456-467.

HARRIS, RJ. 1996. Frequency of overwintered Vespula germanica (Hymenoptera: Vespidae) colonies in scrubland-pasture habitat and their impact on prey. New Zeal. J. Zool., 23:11-17.

Hulme, PE. 2009. Trade, transport and trouble: managing invasive species pathways in an era of globalization. J. Appl. Ecol., 46:10-18.

HuRLEY, BP; B SLIPPERS \& MJ WingFieLd. 2007. A comparison of control results for the alien invasive woodwasp, Sirex noctilio, in the southern hemisphere. Agri. Forest Entomol., 9:159-171.

Lacey, L; R Frutos; H Kaya \& P Vail. 2001. Insect pathogens as biological control agents: do they have a future? Biol. Control, 21:230-248.

LANGor, DW; LJ DeHaAs \& RG FootTit. 2009. Diversity of non-native terrestrial arthropods on woody plants in Canada. Biol. Invasions, 11:5-19.

LANTSChNeR, MV; JM Villacide; JR Garnas; P CROFT; AJ CARNEGIE; AM Liebhold, JC Corley. 2014. Temperature explains variable spread rates of the invasive woodwasp Sirex noctilio in the Southern Hemisphere. Biol. Invasions, 16:329-339.

Liebhold, AM \& PC Tobin. 2008. Population ecology of insect invasions and their management. Annu. Rev. Entomol., 53:387-408.

LiebHold, AM; EG BRockerhofF; LJ GarretT; JL PARKE \& KO BRITTON. 2012. Live plant imports: the major pathway for forest insect and pathogen invasions of the US. Front. Ecol. Environ., 10:135-143.

LOCKWOOD, JL; MF HOOPES \& MP MARCHETTI. 2007. Invasion ecology. Wiley-Blackwell, Oxford.

Lowe, S; M Browne; S Boudjelas \& M De PoOrTer. 2004. 100 of the world's worst invasive alien species a selection from the global invasive species database. The Invasive Species Specialist Group a specialist group of the Species Survival Commission (SSC) of the World Conservation Union (IUCN), Auckland, New Zealand.

MAsCiOCCHI, M \& JC CoRLEY. 2013. Distribution, dispersal and spread of the invasive social wasp (Vespula germanica) in Argentina. Austral Ecol., 38:162-168.

Myers, JH; A SAVOIE \& EV Randen. 1998. Eradication and pest management. Annu. Rev. Entomol., 43:471-491.

SACKMANN, P; M Rabinovich \& JC Corley. 2001. Successful removal of German yellowjackets (Hymenoptera: Vespidae) by toxic baiting. J. Econ. Entomol., 94:811-816.

Sackmann, P \& JC Corley. 2007. Control of Vespula germanica (Hymenoptera, Vespidae) populations using toxic baits: bait attractiveness and pesticide efficacy. J. Appl. Entomol., 131:630-636.

Sharov, AA; D Leonard; AM Liebhold; EA Roberts \& W DICKERSON. 2002. Slow the spread: A national program to contain the gypsy moth. J. Forest., 100:30-36.

Simberloff, D. 2001. Eradication of island invasives: practical actions and results achieved. Trends Ecol. Evol., 16:273-274.

SimberLofF, D. 2009. The role of propagule pressure in biological invasions. Annu. Rev. Ecol. Evol. Syst., 40: 81-102.

Spradbery, JP \& GF Maywald. 1992. The distribution of the European or German wasp, Vespula germanica (F.) (Hymenoptera: Vespidae), in Australia: past, present and future. Aust. J. Zool., 40:495-510.

Tribe, GD \& DM Richardson. 1994. The European wasp, Vespula germanica (Fabricius) (Hymenoptera: Vespidae), in southern Africa and its potential distribution as predicted by ecoclimatic matching. Afr. Entomol., 2:1-6.

VILLACIDE, JM \& JC CoRley. 2003. Distribución potencial del parasitoide Ibalia leucospoides (Hymenoptera: Ibaliidae) en la Argentina. Quebracho. Revista de Ciencias Forestales, 10:7-13.

Villacide, JM \& JC Corley. 2006. Control de niveles poblacionales endémicos de la avispa de los pinos Sirex noctilio (Hymenoptera: Siricidae) mediante el raleo sanitario de hospederos atacados. Rev. Investig. Agropec., 35:121-134.

VILLACIDE, JM \& JC CORLEY. 2012. Ecology of the woodwasp Sirex noctilio: Tackling the challenge of successful pest management. Int. J. Pest Manage., 58:249-256.

Wainhouse, D. 2005. Ecological methods in forest pest management. Oxford Univerity Press, Oxford.

Willink, A. 1980. Sobre la presencia de Vespula germanica (Fabricius) en la Argentina (Hymenoptera: Vespidae). Neotropica, 26:205-206.

Wingfield, M; B Slippers; B Hurley; T Coutinho; B WingFielD; ET AL. 2008. Eucalypt pests and diseases: growing threats to plantation productivity. Southern Forests: a Journal of Forest Science, 70:139-144.

Yemshanov, D; FH Koch; DW McKenney; MC Downing \& F SAPIO. 2009. Mapping Invasive Species Risks with Stochastic Models: A Cross-Border United StatesCanada Application for Sirex noctilio Fabricius. Risk Anal., 29:868-884. 